\title{
Exciton effects in band-edge electroluminescence of silicon barrier structures
}

\author{
A.V. Sachenko*, A.P. Gorban, D.V. Korbutyak, V.P. Kostylyov, \\ Yu.V. Kryuchenko, V.V. Chernenko \\ V. Lashkaryov Institute of Semiconductor Physics, NAS of Ukraine, 45, prospekt Nauky, 03028 Kiev, Ukraine \\ *E-mail: sach@isp.kiev.ua, phone: +380 (44) 2655734
}

\begin{abstract}
A theoretical analysis of the band-edge electroluminescence efficiency in silicon diodes and $p-i-n$-structures has been made. We have shown that maximal possible efficiency can achieve $10 \%$ both at room and liquid nitrogen temperatures. Maximal values of the efficiency are restricted by the interband Auger recombination process. It is found that electroluminescence efficiency decreases rapidly with the decrease of characteristic ShockleyReed-Hall nonradiative lifetime for minority carriers. It is shown that even at room temperatures the main contribution into the edge electroluminescence in silicon barrier structures is given by excitonic effects. Dark I-V characteristics of directly biased silicon diodes measured both at room and nitrogen temperatures are used to explain anomalous temperature dependencies of silicon diode electroluminescence.
\end{abstract}

Keywords: excitons, electroluminescence, internal quantum efficiency, silicon barrier structures.

Paper received 04.11.03; accepted for publication 30.03.04.

\section{Introduction}

In recent years, a substantial interest has grown to the investigations of electroluminescence (EL) in silicon barrier structures at room temperatures (see, for example, [1-3]). This interest is associated, in particular, with possibilities to develop large-scale silicon-based integration circuits with optical coupling elements. Both structures used for photoconversion [1] and semiconductor diodes $[2,3]$ were studied intensively. In $[1,2]$ for the external quantum efficiency of the edge radiation the value of about $1 \%$ was obtained, and in [3] the edge radiation of silicon alloyed diodes was shown to be determined by annihilation of free excitons. Still earlier, the spectral and temperature dependencies of edge electroluminescence were studied in silicon $p-i-n$-structures, and the exciton effects in absorption and radiation were shown to play a substantial role even at room temperatures in these structures too (see, for example, [4]).

In [5-10] the influence of excitons on effective lifetime of electron-hole pairs, edge photoluminescence, I$\mathrm{V}$ characteristics, and efficiency of photoconversion in silicon and silicon barrier structures at room tempera- tures was analyzed. In particular, it was shown that in many cases the effective lifetime of electron-hole pairs is determined by nonradiative exciton Auger recombination with the participation of deep centers, and the internal quantum efficiency of edge photoluminescence can reach the values of about $10 \%$. The analysis of exciton effects in cited works was based on the account of two subsystems of quasiparticles in a semiconductor: electronhole and exciton ones, which are in a quasiequilibrium due to an approximate balance between the number of electron-hole pairs binding into excitons and the number of excitons decomposing into electron-hole pairs per unit time. Using the approach of [5-10], the extreme values of EL quantum efficiencies have been obtained in the present work as functions of structure parameters. We have analysed also temperature dependencies of the intensity and quantum efficiency of the edge EL in silicon barrier structures. An attempt has been made to separate probabilities of exciton and band-to-band radiative recombination. Two types of barrier structures have been considered: diode structures and $p-i-n$-structures, the latter being shown to be promising for optoelectronics applications. 


\section{A.V. Sachenko et al.: Exciton effects in band-edge electroluminescence of silicon ...}

\section{Band-edge electroluminescence in silicon diodes}

Let us consider a case when the thicknesses of $n$ - and $p$ regions exceed diffusion lengths of electrons and holes in these regions. This enables excluding the influence of surface recombination. A linearity over excitation level is also assumed (a case of low-injection conditions is considered in this section), that means that inequalities $n_{n}>>p_{n} \exp (q U / k T)$ and $p_{p}>>n_{p} \exp (q U / k T)$ are realized, where nn and pp are the concentrations of majority charge carriers in $n$ - and $p$-regions, respectively, $p_{n}$ and $n_{p}$ are the concentrations of minority charge carriers in these regions, $q$ is the electron charge (modulo), $k$ is the Boltzman constant, $T$ is the temperature and $U$ is the applied voltage.

The expression for the efficiency of the current-related EL for a long-based diode within the framework of the approach of [5-10] can be written in the following form:

$$
\eta_{J}=\frac{J-J_{0}}{J+J_{r}}
$$

where $J=q\left(D_{p} p_{n} / L_{p}+D_{n} n_{p} / L_{n}\right) \exp (q U / k T)$ is the surface density of the diffusion current in a long-based diode, $J_{r}$ is the recombination current surface density in the space charge regions (SCR), $L_{p}$ and $L_{n}, D_{p}$ and $D_{n}$ are diffusion lengths and coefficients of minority charge carriers, respectively, in $n$ - and $p$-regions. Taking into account the linearity over the excitation level the following expressions for $L_{p}$ and $L_{n}$ can be obtained in accordance with $[5,6]$ :

$$
\begin{aligned}
& L_{p}=\sqrt{D_{p}\left[\frac{1}{\tau_{r p}}+\left(A_{i}+\frac{1}{n^{*} \tau_{x}}\right) n_{n}+\left(C_{n}+C_{p}\right) n_{n}^{2}\right]^{-1}}, \\
& L_{n}=\sqrt{D_{n}\left[\frac{1}{\tau_{r n}}+\left(A_{i}+\frac{1}{n^{*} \tau_{x}}\right) p_{p}+\left(C_{n}+C_{p}\right) p_{p}^{2}\right]^{-1}},
\end{aligned}
$$

where $\tau_{r p}$ and $\tau_{r n}$ are the Shockley-Reed-Hall lifetimes in $n$ - and $p$-regions, respectively, $A_{i}$ is the radiative recombination constant characterising the recombination from continuum electron-hole states correlated by Coulomb interaction [4], $n^{*}=\left(N_{c} N_{v} / N_{x}\right) \exp \left(-E_{x} / k T\right)$, where $N_{c}$, $N_{v}$ and $N_{x}$ are the effective densities of states for electrons, holes and excitons, $E_{x}$ is the exciton binding energy, $\tau_{x}=\left(1 / \tau_{x}^{r}+1 / \tau_{x}^{n}\right)^{-1}$, where $\tau_{x}^{r}$ is the radiative and $\tau_{x}^{n}$ is nonradiative lifetime of excitons, the last being associated with the exciton Auger recombination with deep level participation [11], $C_{n}$ and $C_{p}$ are the parameters of interband electron and hole Auger recombination, respectively. Current density $J_{0}$ is that obtained from the expression for $J$ when terms with $1 / n^{*} \tau_{x}^{r}$ and $A_{i}$ are omitted in Eqs (2) and (3).

If $J_{r}$ is small compared to $J$, and, besides, those terms in square brackets in Eqs (2) and (3) which correspond to the radiative recombination processes are small compared to the terms corresponding to the nonradiative recombination, then expression for current density of the bandedge EL takes up the following form:

$J_{e}=\frac{q}{2} n_{i}^{2}\left(L_{p 0}+L_{n 0}\right)\left(A_{i}+\frac{1}{n^{*} \tau_{x}^{r}}\right)\left[\exp \left(\frac{q U}{k T}\right)-1\right]$,

where $L_{p 0}$ and $L_{n 0}$ are obtained from the expressions for $L_{p}$ and $L_{n}$ if in the latter the terms in square brackets associated with the radiative recombination are omitted. The expression for the internal quantum efficiency of band-edge EL in correspondence with the work [3] takes up the following form

$\eta=n_{n} p_{p} \cdot \frac{L_{p}+L_{n}}{D_{p} p_{p} / L_{p}+D_{n} n_{n} / L_{n}}\left(A_{i}+\frac{1}{n^{*} \tau_{x}^{r}}\right)$.

\section{Band-edge electroluminescence in silicon $p$-i-n-structures}

Let us further obtain an analytical expression for the internal quantum efficiency of the band-edge EL for a silicon $p-i$ - $n$-structure assuming that the $i$-region is weakly doped, and high-injection conditions are realised, so that the criterion $n_{n} \ll p_{n} \exp (q U / k T)$ or $p_{p}<<n_{p} \exp (q U / k T)$ is met. Besides, we will consider that the i-region thickness is smaller than the hole or electron diffusion length. By neglecting the recombination of electron-hole pairs at the surfaces of $p^{+}$- and $n^{+}$-regions and the recombination current in SCR compared to the diffusion one, the internal quantum efficiency of the EL in accordance with [8] can be written in the following form:

$\eta=$

$$
=\frac{A_{i}+\frac{1}{n^{*} \tau_{x}^{r}}}{\left(\frac{1}{n_{i} \tau_{r}}\right) \exp \left(-\frac{q U}{2 k T}\right)+A_{i}+\frac{1}{n^{*} \tau_{x}}+\left(C_{n}+C_{p}\right) n_{i} \exp \left(\frac{q U}{2 k T}\right)},
$$

where $\tau_{r}$ is the Shokley-Reed-Hall time at high-injection conditions, and $n_{i}$ is the charge carrier concentration in intrinsic silicon. The current density of EL in this case is equal to

$J_{e}=q d\left(A_{i}+\frac{1}{n^{*} \tau_{x}^{r}}\right) n_{i}^{2} \exp \left(\frac{q U}{k T}\right)$.

Noteworthy is that in the case under consideration the excess concentration of electron-hole pairs $\Delta p$ is determined by the relation

$\Delta p=n_{i} \exp (q U / 2 k T)$ 


\section{A.V. Sachenko et al.: Exciton effects in band-edge electroluminescence of silicon ...}

\section{Temperature dependencies of exciton electroluminescence in silicon barrier structures}

Let us first analyse the temperature dependencies of quantum efficiency and intensity of the exciton EL for longbased diodes. Taking into account that the radiative exciton recombination lifetime changes with temperature as $\sim T^{3 / 2}$, it follows from the expressions (1)-(5) that in the cases, when in the nonradiative recombination dominates (a) recombination of Shockley-Reed-Hall, (b) exciton Auger recombination, or (c) interband Auger recombination, the following relationships are valid for the internal quantum efficiencies $\eta_{x}$ and current densities $J_{e x}$ of the exciton EL:

$\eta_{x} \sim T^{-3 / 2} \tau_{r}(T) \exp \left(E_{x} / k T\right)$,

$\eta_{x} \sim T^{-3 / 2} \tau_{r}(T)$

$\eta_{x} \sim T^{-3 / 2} \exp \left(E_{x} / k T\right) /\left[C_{j}(T) n_{j}^{2}\right]$,

$J_{e x} \sim \sqrt{D(T) \tau_{r}(T) T^{-3}} \exp \left(E_{x} / k T\right)$,

$J_{e x} \sim \sqrt{D(T) \tau_{r}(T) T^{-3}} \exp \left(E_{x} / 2 k T\right)$,

$J_{e x} \sim \sqrt{D(T) T^{-3} /\left[C_{j}(T) n_{j}^{2}\right]} \exp \left(E_{x} / k T\right)$,

where $n_{j}=n_{n}$ for the $n$-regions and $n_{j}=p_{p}$ for $p$-regions

As numerical estimates show, the exciton EL current density of silicon diodes in all the limiting cases mentioned increases with temperature decrease. In the case (a) the exciton EL internal quantum efficiency increases with temperature drop as well. However, more topical are the cases (b), when the nonradiative recombination is determined by the exciton Auger recombination, and (c), when the interband Auger recombination dominates. In the case (b) everything depends on how the ShockleyReed-Hall lifetime $\tau_{r}(T)$ changes with temperature, while in the case (c) on whether the exciton radiative recombination or the interband Auger recombination grows faster with temperature drop. For deep recombination centers in silicon $\tau_{r}$ first decreases to a certain value with temperature decrease from the room temperature, and then ceases to change. In this region of practical independence of $\tau_{r}$ from the temperature the exciton EL internal quantum efficiency must grow with the temperature decrease as $T^{-3 / 2}$. Just the same behaviour of EL quantum efficiency has to be observed also in $p$ - $i$-n-structures when the case (b) is realised. Under the condition $n_{i}(T) \exp (q U / 2 k T)=\Delta n=$ $=$ const , the exciton EL current density for $p-i-n$-structures is equal to

$J_{e x}=q d \frac{(\Delta n)^{2}}{n^{*} \tau_{x}^{r}}$

and depends on temperature only due to temperature dependencies of $n^{*}$ and $\tau_{x}^{r}$. In this case, $J_{e x}$ grows with the temperature decrease stronger than $T^{-3 / 2}$.

The results of the above theoretical analysis contradict to the experimental data on anomalous temperature dependence of the EL intensity in alloyed silicon diodes presented in [3]. To elucidate the reason for this discrepancy, we have measured dark I-V curves on a number of standard alloyed silicon diodes at room temperature and at liquid nitrogen temperature. The results on dark I-V measurements for one of such diodes are shown in Fig. 1. The I-V non-ideality factor for these diodes in the region of rather large currents amounted to about 1.5 at room temperature and 2.24 at liquid nitrogen temperature. This means that under condition of constant total current the contribution of the diffusion current component at $T=80 \mathrm{~K}$ is substantially smaller than at room temperature. At the same time, the exciton EL is associated only with the dark diffusion current component because all other mechanisms of current flow are strongly field-dependent, and in the region of strong fields $\geq 10^{5} \mathrm{~V} / \mathrm{cm}$ excitons are practically absent in silicon. Therefore, it is clear that to determine correctly the temperature dependence of EL intensity in the case of alloyed silicon diodes, one needs to measure not the total dark current, but only its diffusion component. The modern silicon diffusion diodes have

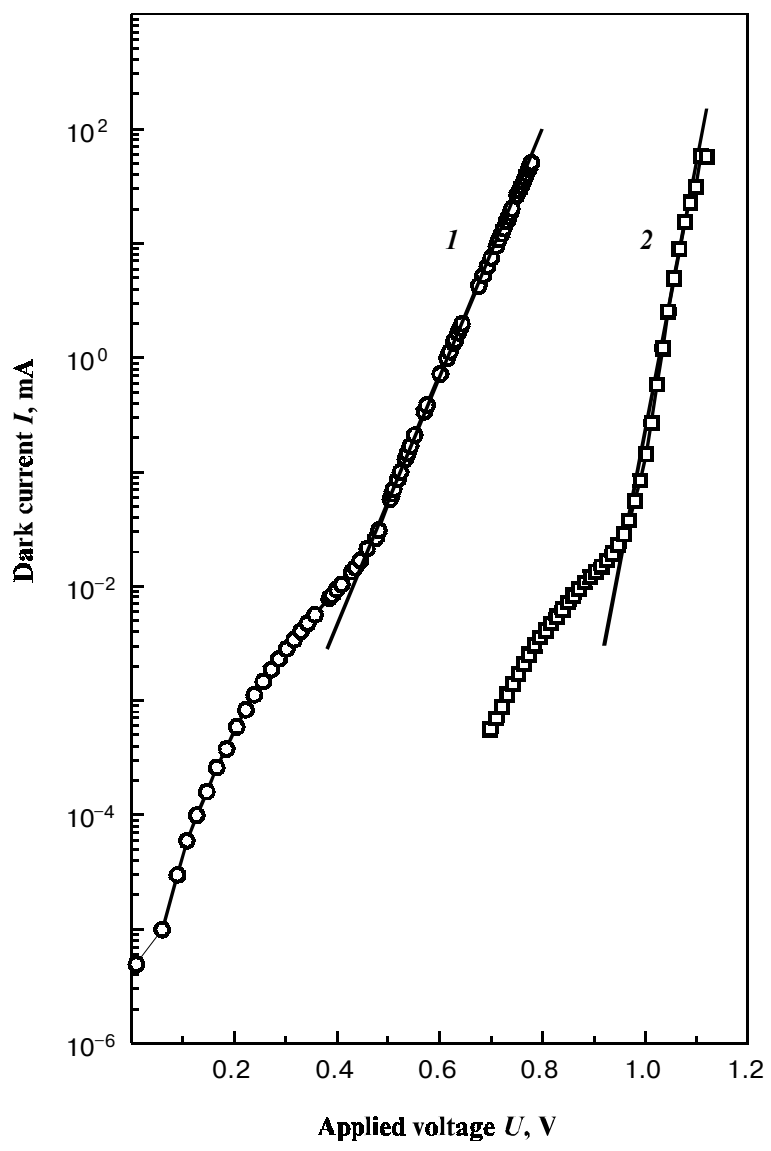

Fig. 1. Dark $\mathrm{I}-\mathrm{V}$ characteristics of an alloyed silicon diode. Curves: $1-T=300 \mathrm{~K}, 2-T=77 \mathrm{~K}$ 


\section{A.V. Sachenko et al.: Exciton effects in band-edge electroluminescence of silicon ...}

nonideality factor close to unity (see e.g. [1]) and the above discussed problems are absent at all.

\section{Separation of electron-hole and exciton radiative recombination contributions}

Although attempts to separate contributions of band-toband and exciton radiative recombinations in silicon were made already earlier, the problem is still far from its final solution. While considering it in the present work, we will suggest that two subsystems coexist in silicon, namely subsystem of free electron-hole pairs correlated by coulomb interaction and subsystem of bound electron-hole states, i.e. excitons. The quasi-equilibrium between them is described by the thermodynamic relations. Such an approach is true for the region of not too low temperatures when the criterion $n_{0} \exp \left(-E_{x} / k T\right)>>1 / \gamma_{1} \tau_{x}$ is met, where $\gamma_{1}$ is the probability of electron-hole pair binding into excitons. In this case the total probability of the radiative quadratic recombination can be written as $A_{i}+1 / n^{*} \tau_{x}^{r}$, where $A_{i}$ is the probability of the radiative band-to-band recombination, and $1 / n^{*} \tau_{x}^{r}$ is the probability of the radiative exciton recombination. At room temperature their total value amounts to approximately 2.5 $10^{-15} \mathrm{~cm}^{3} / \mathrm{s}$ [5]. Temperature dependencies of this value obtained in different approximations are given in [4]. Separation of band-to-band and exciton contributions is associated with the following difficulties. Firstly, the value of $1 / n^{*} \tau_{x}^{r}$ may substantially depend on the equilibrium or excess concentration of electrons or/and holes due to screening of Coulomb interaction between electrons and holes by mobile charge carriers. Secondly, in this case it is necessary to know the exact value of the intrinsic charge carrier concentration $n_{i}$ in silicon accounting for the temperature dependencies of the density-of-state effective masses in the conduction and valence bands $[12,13]$.

Due to effect of screening of Coulomb interaction between electron and hole, the probability of the exciton radiative recombination strongly decreases while approaching the exciton Mott transition (especially at low temperatures) down to zero at critical and higher concentrations of free carriers. This can occur both at low injection levels due to increase of $\mathrm{p}$ - and n-regions doping and at high injection levels due to increase of the applied voltage. In this work, the analysis and separation of probabilities of band-to-band and exciton radiative recombinations have been carried out by accounting for the above peculiarities.

In a general case, we used the following expression for $n^{*}[6]$ :

$$
\begin{aligned}
& n^{*}=1.208 \cdot 10^{15}\left(T \cdot m_{x}^{*}(T) / m_{0}\right)^{3 / 2} \times \\
& \times \exp \left[0.567\left(\frac{300}{T}\right)\left(1-\left(\frac{n_{n}+2 \Delta n}{8.3 \cdot 10^{17}(T / 300)}\right)^{1 / 2}\right)^{2}\right],
\end{aligned}
$$

where $m_{x}^{*}(T) / m_{0}$ is the ratio of the normalized densityof-state effective mass for an exciton in silicon to the mass of a free electron taking into account temperature dependencies of density-of-state effective masses for electron and hole $[12,13]$. Numerical values of the concentrations in this and subsequent formulae have to be substituted in $\mathrm{cm}^{-3}$ units.

As mentioned above, the total probability of edge band-to-band and exciton recombinations $A_{i}+1 / n^{*} \tau_{x}^{r}$ obtained in [5] from the dependence of electron-hole pair effective lifetimes in silicon on the excitation level amounts to $2.5 \cdot 10^{-15} \mathrm{~cm}^{3} / \mathrm{s}$ at room temperature. This value coincides with both theoretical value obtained in [13], where the principle of detailed equilibrium between absorption and radiation was employed (the van RoosbroeckShockley approach), and experimental value obtained in [14] from spectral dependence of light absorption coefficient in silicon near the intrinsic absorption edge.

Using the data of [15], we have calculated in a similar way the value $A_{i}+1 / n^{*} \tau_{x}^{r}=7.22 \cdot 10^{-14} \mathrm{~cm}^{3} / \mathrm{s}$ at $T=90 \mathrm{~K}$. In turn, this value practically coincides with the microscopic theory result obtained in [4]. As the temperature dependence of the radiative electron-hole recombination parameter $A_{i}$ is rather weak $\left(\sim T^{-1 / 2}\right.$, see for instance [16]), and at $T=300 \mathrm{~K}$ the $A_{i}$ value cannot be higher than $2.5 \cdot 10^{-15} \mathrm{~cm}^{3} / \mathrm{s}$, it is clear that the total radiative recombination probability at $T=90 \mathrm{~K}$ is in fact determined by the second term $1 / n^{*} \tau_{x}^{r}$, which corresponds to the radiative exciton recombination. Using Eq. (12) and choosing $\tau_{x}^{r}=10^{-3}$ s at $T=300 \mathrm{~K}$, suggesting, as earlier, that the exciton radiative lifetime $\tau_{x}^{r}(T)$ changes with temperature as $T^{3 / 2}$, we obtain for the doping level $7 \cdot 10^{15} \mathrm{~cm}^{-3}$ at which the absorption coefficient was measured the value $1 / n^{*} \tau_{x}^{r}=7.0 \cdot 10^{-14} \mathrm{~cm}^{3} / \mathrm{s}$ at $T=90 \mathrm{~K}$, which is in good agreement with the above results. At $300 \mathrm{~K}$ the exciton radiative recombination probability $1 / n^{*} \tau_{x}^{r}$ calculated with the parameters indicated above amounts to $6.1 \cdot 10^{-16} \mathrm{~cm}^{3} / \mathrm{s}$. This means that the value $1.9 \cdot 10^{-15} \mathrm{~cm}^{3} / \mathrm{s}$ remaining after subtraction of $6.1 \cdot 10^{-16} \mathrm{~cm}^{3} / \mathrm{s}$ from $2.5 \times 10^{-15} \mathrm{~cm}^{3} / \mathrm{s}$ really is the sum of $A_{i}$ and corresponding contribution into the radiative recombination of conduction and valence bands states correlated by Coulomb interaction [4]. Since theoretically calculated value of $A_{i}$ in silicon using formulae of [16] and temperature dependencies of the density-of-state effective masses for electrons and holes $[12,13]$ is about $8 \cdot 10^{-16} \mathrm{~cm}^{3} / \mathrm{s}$ at $T=300 \mathrm{~K}$, it is clear that according to our estimates the part of radiative recombination probability in silicon which is determined by exciton transitions and transitions in a subsystem of correlated electron-hole band states at room temperature does not exceed $1.7 \cdot 10^{-15} \mathrm{~cm}^{3} / \mathrm{s}$.

\section{Numerical calculations of the internal quantum efficiency of electroluminescence in silicon barrier structures}

As was shown in [17], in the region of intermediate charge carrier concentrations, from $10^{15}$ to $10^{18} \mathrm{~cm}^{-3}$, the coeffi- 


\section{A.V. Sachenko et al.: Exciton effects in band-edge electroluminescence of silicon ...}

cient $C_{n}$ of interband Auger recombination in silicon with electron participation strongly depends on electron-electron and electron-hole Coulomb interaction and substantially exceeds the values typical for the case of high carrier concentrations when the Coulomb interaction is strongly screened. In [5] empirical approximations were obtained for the $C_{n}$ values determined from the experimental data of [17] and calculated theoretically. These approximations look like

$C_{n}=\left(2.3 \cdot 10^{-31}+\frac{1.3 \cdot 10^{-12}}{n_{n}+\Delta n}\right) \mathrm{cm}^{6} \mathrm{~s}^{-1}$

and

$C_{n}=\left(2.8 \cdot 10^{-31}+\frac{2.5 \cdot 10^{-22}}{\left(n_{n}+\Delta n\right)^{1 / 2}}\right) \mathrm{cm}^{6} \mathrm{~s}^{-1}$,

respectively.

As our analysis has shown, just the interband Auger recombination confines the EL internal quantum efficiency of silicon barrier structures in the case of extremely large Shockley-Reed-Hall recombination times, $\tau_{r} \geq 10^{-3} \mathrm{~s}$. Calculations have shown that for $\tau_{r}=4 \cdot 10^{-2} \mathrm{~s}$ the maximal EL internal quantum efficiency in silicon at room temperatures amounts to about $10 \%$. To calculate $\eta$ at $70 \mathrm{~K}$ we need to know the temperature dependence of $\tau_{r}$. In many cases the Shockley-Reed-Hall recombination time first decreases with the temperature decrease and then ceases to depend on temperature $[18,19]$. It was shown, in particular, in [20], that with temperature de- crease from room to liquid nitrogen this parameter decreases in silicon of n-type by a factor of 5. For numerical calculations we used the empirical dependence $\tau_{r}(T)=\tau_{r}(300)\left(T / 300+10^{-1}\right)^{3 / 2}$, which gives approximately the same decrease of $\tau_{r}$. The calculations made with account for above-mentioned effects show that at $T=70 \mathrm{~K}$ the extreme values of EL internal quantum efficiency both in silicon diodes and $p-i$ - $n$-structures is somewhat greater than $10 \%$.

In Fig. 2, which illustrates our results for silicon diodes with different doping levels of $p$-type region, the dependencies of $\eta$ on the doping level of $n$-type region are built for the temperatures 300 and $70 \mathrm{~K}$. It is seen from the figures that maximal EL quantum efficiency is approximately the same at both temperatures. In both cases it is limited by the interband Auger recombination. This recombination provides some decrease of $\eta$ with the growth of doping level of the diode $n$-region.

In Fig. 3, the dependencies of $\eta$ on the applied voltage are built for $p$ - $i$ - $n$-structures with a short base at 300 and $70 \mathrm{~K}$. Like in the case of diodes, the extreme values of $\eta$ are limited by the interband Auger recombination. At the same time, at small enough Shockley-Reed-Hall lifetimes, $\tau_{r} \leq 10^{-4} \mathrm{~s}$, a limiting case (c), when nonradiative exciton recombination dominates, is realized. That is why the EL internal quantum efficiency remains constant within a certain range of applied voltages.

It should be noted that the extreme $\eta$ values at $T=70 \mathrm{~K}$ are somewhat lower than those at $T=300 \mathrm{~K}$ although the situation seemed to be opposite. This occurs
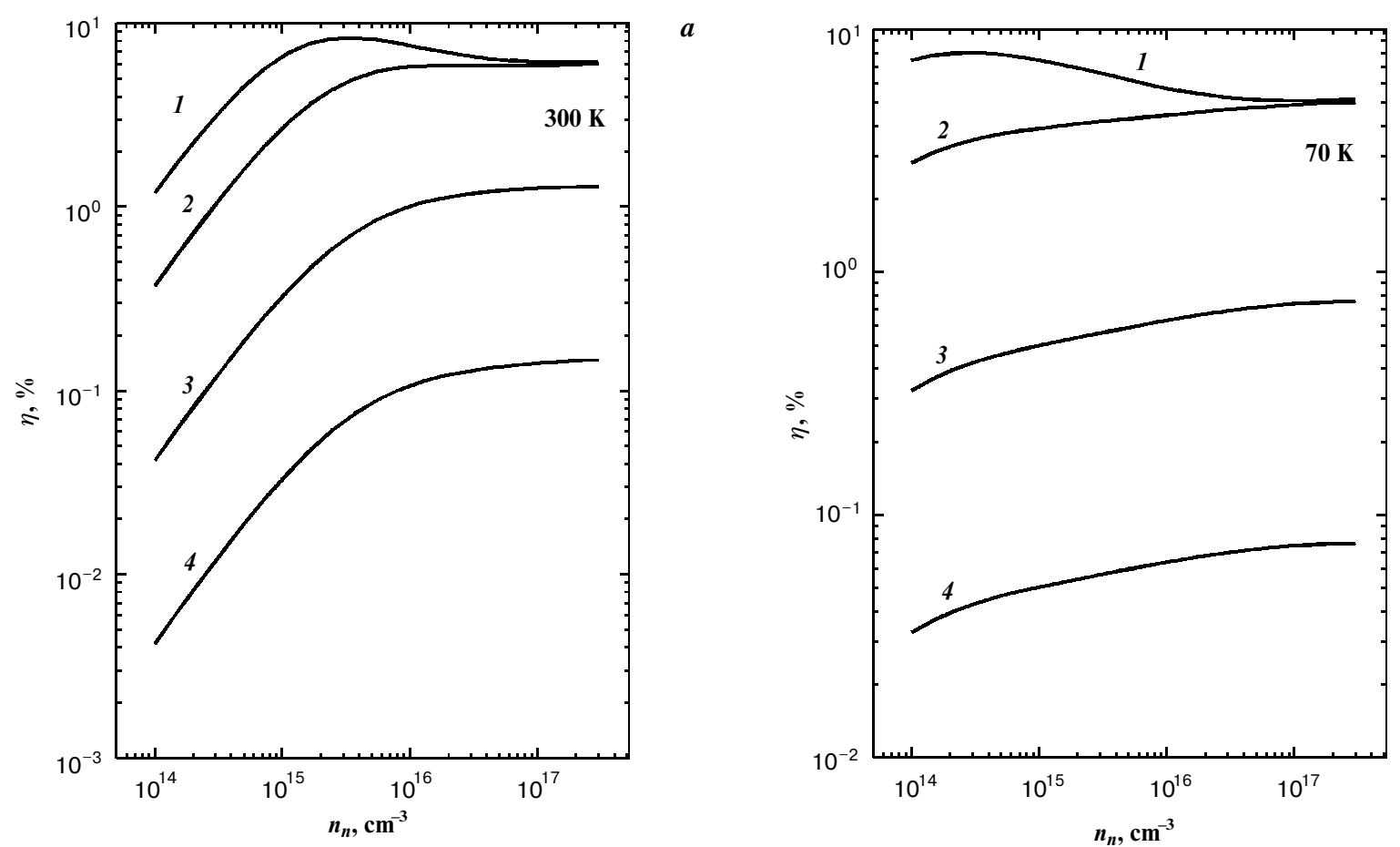

Fig. 2. The dependencies of the EL internal quantum efficiency of silicon diodes on the doping level of $n$-type region at $\mathrm{T}=300 \mathrm{~K}$ and $T=70 \mathrm{~K}$. Characteristic Shockley-Reed-Hall nonradiative lifetime $\tau_{r p}$ and $\tau_{r n}$ at $T=300 \mathrm{~K}: 1-4 \cdot 10^{-2}$ and $7 \cdot 10^{-3} ; 2-10^{-2}$ and $7 \cdot 10^{-3} ; 3-$ $10^{-3}$ and $10^{-3} ; 4-10^{-4}$ and $10^{-4} \mathrm{~s}$. The concentrations of majority charge carriers in $p$-region equal to $3 \cdot 10^{16}(T=300 \mathrm{~K})$ and $2 \cdot 10^{15} \mathrm{~cm}^{-3}$ $(T=70 \mathrm{~K})$. 
A.V. Sachenko et al.: Exciton effects in band-edge electroluminescence of silicon ...
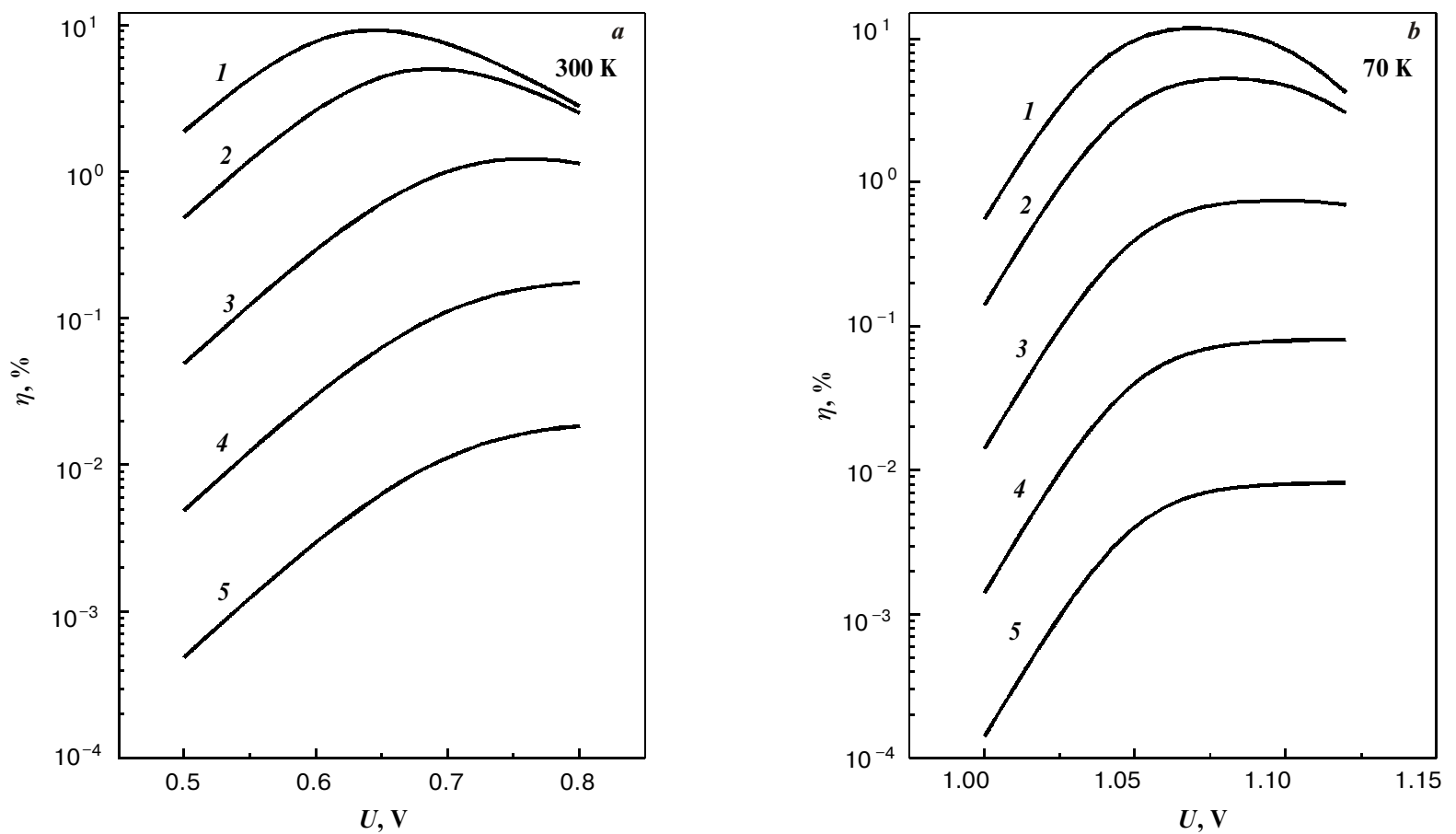

Fig. 3. The dependencies of the EL internal quantum efficiency of silicon $p$-i-n-structures on applied voltage at $T=300 \mathrm{~K}$ and $T=70 \mathrm{~K}$. Characteristic Shockley-Reed-Hall nonradiative lifetime $\tau_{r}$ at $T=300 \mathrm{~K}: 1-4 \cdot 10^{-2}, 2-10^{-2}, 3-10^{-3}, 4-10^{-4}, 5-10^{-5} \mathrm{~s}$.

due to strong influence of Coulomb interaction on the interband Auger recombination probability in silicon. As a result, at not too high charge carrier concentrations the Auger recombination probability grows stronger with temperature decrease than the radiative exciton recombination probability.

In the range of rather low temperatures, when the Shockley-Reed-Hall lifetime ceases to change with temperature, we still may hope for a substantial increase in the EL internal quantum efficiency in silicon barrier structures compared to the case of room temperatures, especially, at comparatively low $\tau_{r}$ values.

\section{Conclusions}

Thus, the theoretical analysis carried out in the present work has shown that even at room temperature the exciton effects in the radiative recombination give the main contribution into the band-edge EL of silicon barrier structures. With temperature decrease the contribution of radiative exciton recombination into the total band-edge electroluminescence grows sharply and becomes dominant, i.e. determines latter practically completely.

The obtained results indicate that under the extreme quantum efficiency the silicon diodes are close to the $p$-i-n-structures. However, to achieve maximum efficiency in silicon diodes, their thickness should be not smaller than $1 \mathrm{~cm}$, i.e. be equal to or exceed the sum of the diffusion lengths in $p$ - and $n$-regions with maximal achieved Shockley-Reed-Hall lifetimes, while in the case of $p-i-n$ - structures the extreme values of EL quantum efficiency does not depend on the base thickness. For this reason, the electroluminescent silicon $p$ - $i-n$-structures, to our mind, are promising for optoelectronics applications.

\section{References}

1. M.A. Green, J. Zhao, A. Wang, P. J. Reece, M. Gal. Efficient silicon light-emitting diodes // Nature, 412, pp. 805-808 (2001).

2. W.L. Ng, M.A. Lourenco, R.M. Gwilliam, S. Lewdain, G. Shao, K.P. Homewood. An efficient room-temperature silicon-based light-emitting duiode // Nature, 410, pp. 192194 (2001).

3. M.S. Bresler, O.B. Gusev, B.P. Zakharchenya, I.N. Yassievich. Electroluminescence efficiency of silicon diodes // in Materials of Symposium "Nanophotonics" (Nizhnii Novgorod, Russia, 2003), vol. 1, pp. 59-62 (in Russian).

4. M. Ruff, M. Fick, R. Lindner, U. Rossler, R. Helbig. The spectral distribution of the intrinsic radiative recombination in silicon // J. Appl. Phys., 74(1), pp. 267-274 (1993).

5. A.V. Sachenko, A.P. Gorban, V.P. Kostylyov. Exciton-enhanced recombination in silicon at high concentrations of charge carriers // Semiconductor Physics, Quantum Electronics \& Optoelectronics, 3(1), pp. 5-10 (2000).

6. A.V. Sachenko, Yu V. Kryuchenko, Exciton effects in bandedge luminescence of semiconductors at room temperatures // Semiconductor Physics, Quantum Electronics \& Optoelectronics, 3(2), pp. 150-156 (2000).

7. A.P. Gorban, A.V. Sachenko, V.P. Kostylyov, N.A. Prima. Effect of excitons on photoconversion efficiency in the $\mathrm{p}+-\mathrm{n}-$ $n+$ and $n+-p-p+-$ structures based on single-crystalline silicon // Semiconductor Physics, Quantum Electronics \& Optoelectronic, 3(3), pp. 322-329 (2000). 


\section{A.V. Sachenko et al.: Exciton effects in band-edge electroluminescence of silicon ...}

8. A.V. Sachenko, A.P. Gorban, V.P. Kostylyov. Exciton mechanism of recombination in silicon and its influence on physical cheracteristics of semiconductor structures (review) // Ukrainian Journal of Physics, 46(2), pp. 226 -239 (2001) (in Ukrainian)

9. A.V. Sachenko, N. A. Prima, A. P. Gorban, A. A. Serba Effect of excitons on the upper limit of conversion efficiency in silicon solar cells // in Proc. 17-th European Photovoltaic Solar Energy Conference (Munich, Germany, 2001), vol. 1, p. 230-233 (2001).

10. A.P. Gorban, V.P. Kostylyov, A.V. Sachenko, V.V. Chernenko. Generalised analytical model for calculation of conversion efficiency in silicon solar cells // in. Proc. 17-th European Photovoltaic Solar Energy Conference (Munich, Germany, 2001), vol. 1, p. 234-237 (2001).

11. A. Hangleiter. Nonradiative recombination via deep impurity levels in silicon: Experiment // Phys. Rev.B., 35(17), pp.9149-9160 (1987).

12. M.A. Green. Intristic concentration, effective densities of states, and efective mass in silicon. Appl. Phys., 67(6), pp. 2944-2955(1990).

13. A.P. Gorban, V.A. Zuev, V.P. Kostylyov, A.V. Sachenko, A.A. Serba, V.V. Chernenko. On temperature dependences of equilibritum and non-equilibritum characterisitic in silicon // Optoelectronics and Semiconductor Technics, No $\mathbf{3 6}$, pp. 161-164 (2001) (in Russian).
14. M.J. Keevers, M.A. Green. Optical properties of intrinsic silicon at $300 \mathrm{~K} / /$ Appl. Phys. Lett., 66, 174 (1995).

15. A. Neisser and M.A. Green. Very low absorption coefficients of silicon at low temperatures from spectral response measurements // in Proc. Second World Conference and Exhibition on Photovoltaic Solar Energy Conversion (Vienna, Austria, 1998), p. 136-139 (1998).

16. H. Schlangenotto, H. Maeder, and W. Gerlach. Temperature dependence of the radiative recombination coefficient in silicon // Phys. Stat. Sol., (a), 21(1), pp. 357-367 (1974).

17. A. Hangleiter and R. Hacker, Intrinsic upper limits of the carrier lifetime in silicon // Phys. Rev. Lett., 65(11), pp.450453(1990).

18. K.D. Glinchuk, N.M. Litovchenko, Recombination centers in thermally treated silicon // Optoelectronics and Semiconductor Technics, No 7, pp. 58-69 (1985) (in Russian).

19. V.N. Abakumov, V.I. Perel, I.N. Yassievich, in: Modern Problems in Condenced Matter Sciences series, vol. 33, edited by V.M. Agranovich and A.A. Maradudin (North-Holland, Amsterdam) (1991).

20. R. Hacker and A. Hangleiter. Intrinsic upper limits of the carrier lifetime in silicon // J. Appl. Phys., 75(11), pp. 75707572 (1994). 